\title{
Weighted integral representations of harmonic functions in the unit disc by means of Mittag-Leffler type kernels
}

\author{
F. V. Hayrapetyan
}

Abstract. For weighted $L^{p}$-classes of functions harmonic in the unit disc, we obtain a family of weighted integral representations with weight function of the type $|w|^{2 \varphi} \cdot\left(1-|w|^{2 \rho}\right)^{\beta}$.

Key Words: Harmonic Functions in the Unit Disc, Weighted Function Space, Weighted Integral Representation

Mathematics Subject Classification 2010: 30F15, 30C40, 30H20, 30E20

\section{Introduction}

It is well-known that the Cauchy integral formula has numerous applications in complex analysis. This formula makes it possible to reproduce values of holomorphic functions inside of a domain by integration of function along the boundary of the domain. First results are contained in [1, 2], where the values of holomorphic functions inside of a domain were obtained by integration of functions over the whole domain. In [3, 4] for the weighted spaces $H^{p}(\alpha)(1 \leq p<\infty, \alpha>-1)$ of functions $f$ holomorphic in the unit disc $\mathbb{D}$ and satisfying the condition

$$
\int_{\mathbb{D}}|f(\zeta)|^{p}\left(1-|\zeta|^{2}\right)^{\alpha} d u d v<+\infty, \quad \zeta=u+i v,
$$

the following result was established:

Theorem 1. Each function $f \in H^{p}(\alpha)$ has the integral representation

$$
\begin{aligned}
& f(z)=\frac{\alpha+1}{\pi} \int_{\mathbb{D}} \frac{f(\zeta)\left(1-|\zeta|^{2}\right)^{\alpha}}{(1-z \bar{\zeta})^{2+\alpha}} d u d v, \quad z \in \mathbb{D}, \\
& \overline{f(0)}=\frac{\alpha+1}{\pi} \int_{\mathbb{D}} \frac{\overline{f(\zeta)}\left(1-|\zeta|^{2}\right)^{\alpha}}{(1-z \bar{\zeta})^{2+\alpha}} d u d v, \quad z \in \mathbb{D} .
\end{aligned}
$$


These representations had numerous applications in the theory of factorization of meromorphic functions in the unit disc (see [3, 4] as well as [5]).

Assume that $0<p<+\infty, \rho>0, \alpha>-1, \gamma>-1$. Denote by $L_{\alpha, \rho, \gamma}^{p}(\mathbb{D})$ the set of all complex-valued measurable functions $f(\zeta), \zeta \in \mathbb{D}$, for which

$$
M_{\alpha, \rho, \gamma}^{p}(f) \equiv \iint_{\mathbb{D}}|f(\zeta)|^{p}\left(1-|\zeta|^{2 \rho}\right)^{\alpha}|\zeta|^{2 \gamma} d m(\zeta)<+\infty .
$$

Also we will use the following notations:

$$
\begin{gathered}
H_{\alpha, \rho, \gamma}^{p}(\mathbb{D})=\left\{f \in H(\mathbb{D}): M_{\alpha, \rho, \gamma}^{p}(f)<+\infty\right\}, \\
h_{\alpha, \rho, \gamma}^{p}(\mathbb{D})=\left\{f \in h(\mathbb{D}): M_{\alpha, \rho, \gamma}^{p}(f)<+\infty\right\},
\end{gathered}
$$

where $H(\mathbb{D})$ and $h(\mathbb{D})$ are the sets of all holomorphic and harmonic functions in $\mathbb{D}$, respectively.

The spaces above were introduced in [6]. Moreover, for these spaces an analogue of representations (1) and (2) were written out by means of special reproducing kernels adapted to new weight functions (see [6] and [7]):

Let $\rho>0, \operatorname{Re} \beta>-1, \operatorname{Re} \varphi>-1$ and $\mu=\frac{1+\varphi}{\rho}$. Then put

$$
S_{\beta, \rho, \varphi}(z, \zeta)=\frac{\rho}{\pi \Gamma(\beta+1)} \sum_{k=0}^{\infty} \frac{\Gamma\left(\mu+\beta+1+\frac{k}{\rho}\right)}{\Gamma\left(\mu+\frac{k}{\rho}\right)} z^{k} \bar{\zeta}^{k},
$$

where $z \in \mathbb{D}$ and $\zeta \in \overline{\mathbb{D}}$.

The main properties of the kernel $S_{\beta, \rho, \varphi}(z, \zeta)$ can be summarized in the following theorem (see [6], [7]).

Theorem 2. 1. For all $z \in \mathbb{D}$ and $\zeta \in \overline{\mathbb{D}}$, the series $S_{\beta, \rho, \varphi}(z, \zeta)$ is absolutely convergent.

2. For all $z \in \mathbb{D}$ and $\zeta \in \overline{\mathbb{D}}$,

$$
\left|S_{\beta, \rho, \varphi}(z, \zeta)\right| \leq \frac{\operatorname{const}(\beta, \rho, \varphi)}{(1-|z|)^{2+R e \beta}}
$$

3. $S_{\beta, \rho, \varphi}(z, \zeta)$ can be majorated by a positive convergent series uniformly in $z \in K \subset \mathbb{D}$ and $\zeta \in \overline{\mathbb{D}}$, where $K$ is a compact set.

4. For a fixed $\zeta \in \overline{\mathbb{D}}, S$ is holomorphic in $z \in \mathbb{D}$. For a fixed $z \in \mathbb{D}, S$ is antiholomorphic in $\zeta \in \mathbb{D}$ and continuous in $\zeta \in \overline{\mathbb{D}}$. 
5. For all $z \in \mathbb{D}$ and $\zeta \in \overline{\mathbb{D}}$,

$$
S_{\beta, \rho, \varphi}(z, \zeta)=\frac{\rho}{\pi \Gamma(\beta+1)} \int_{0}^{\infty} e^{-t} t^{\mu+\beta} E_{\rho}\left(t^{\frac{1}{\rho}} z \bar{\zeta} ; \mu\right) d t,
$$

where $E_{\rho}(\cdot ; \mu)$ is the well-known Mittag-Leffler type entire function. Moreover, the function under the sign of the integral is majorated by a positive integrable function uniformly in $z \in K \subset \mathbb{D}$ and $\zeta \in \overline{\mathbb{D}}$, where $K$ is a compact set.

The corresponding generalization of (1) and (2) is formulated as follows (see [6], [7]):

Theorem 3. Assume that $1 \leq p<+\infty, \rho>0, \alpha>-1, \gamma>-1$, complex numbers $\beta$ and $\varphi$ are satisfying the conditions

$$
\operatorname{Re} \beta \geq \alpha, \quad \operatorname{Re} \varphi \geq \gamma
$$

when $p=1$ and the conditions

$$
\operatorname{Re} \beta>\frac{\alpha+1}{p}-1, \quad \operatorname{Re} \varphi>\frac{\gamma+1}{p}-1,
$$

when $p>1$, and put $\mu=(\varphi+1) / \rho$. Then each function $f \in H_{\alpha, \rho, \gamma}^{p}(\mathbb{D})$ has the following representation:

$$
f(z)=\iint_{\mathbb{D}} f(\zeta) S_{\beta, \rho, \varphi}(z, \zeta)\left(1-|\zeta|^{2 \rho}\right)^{\beta}|\zeta|^{2 \varphi} d m(\zeta), \quad z \in \mathbb{D}
$$

and

$$
\overline{f(0)}=\iint_{\mathbb{D}} \overline{f(\zeta)} S_{\beta, \rho, \varphi}(z, \zeta)\left(1-|\zeta|^{2 \rho}\right)^{\beta}|\zeta|^{2 \varphi} d m(\zeta), \quad z \in \mathbb{D} .
$$

In the present paper, we prove an analogous result of the theorem above for harmonic functions from the corresponding weighted $L^{p}$-spaces in the unit disc $\mathbb{D}$.

\section{$2 \quad$ Necessary Estimates}

First of all, we intend to strengthen some assertions of the Theorem 2 .

Theorem 4. Assume $\rho>0, \operatorname{Re} \beta>-1, \operatorname{Re} \varphi>-1, \mu=\frac{1+\varphi}{\rho}, z \in \mathbb{D}, \zeta \in \overline{\mathbb{D}}$ and the kernel $S_{\beta, \rho, \varphi}(z, \zeta)$ is defined by (3). If

$$
\begin{array}{lll}
-1<a_{1} \leq \operatorname{Re} \beta \leq a_{2}<+\infty, & & |\operatorname{Im} \beta| \leq A<+\infty, \\
-1<b_{1} \leq \operatorname{Re} \varphi \leq b_{2}<+\infty, & & |\operatorname{Im} \varphi| \leq B<+\infty
\end{array}
$$


and $\zeta \in \overline{\mathbb{D}},|z| \leq \lambda<1$, then the expression of $S_{\beta, \rho, \varphi}$ can be uniformly majorated by the convergent series

$$
\operatorname{const}\left(a_{1}, a_{2}, A, b_{1}, b_{2}, B, \rho\right) \sum_{k=0}^{\infty}(k+1)^{a_{2}+1} \lambda^{k} .
$$

Moreover,

$$
\left|S_{\beta, \rho, \varphi}(z, \zeta)\right| \leq \frac{\operatorname{const}\left(a_{1}, a_{2}, A, b_{1}, b_{2}, B, \rho\right)}{(1-|z||\zeta|)^{2+R e \beta}} \leq \frac{\operatorname{const}\left(a_{1}, a_{2}, A, b_{1}, b_{2}, B, \rho\right)}{(1-\lambda)^{2+a_{2}}} .
$$

Proof. According to Stirling formula (see, for example, 8], pp. 158-159) there exist numbers $a, b, 0<a<b<+\infty$, such that

$$
a \leq \frac{|\Gamma(\mu+R)|}{R^{R e \mu+R-1 / 2} \cdot e^{-R}} \leq b
$$

uniformly in $\mu \in K \Subset\{\mu \in \mathbb{C}: \operatorname{Re} \mu>0\}$ and $0<\delta \leq R<+\infty$. Hence, in view of (6),

$$
\begin{aligned}
& \left|S_{\beta, \rho, \varphi}(z, \zeta)\right| \leq \frac{\rho}{\pi} \frac{|\Gamma(\mu+\beta+1)|}{|\Gamma(\beta+1)||\Gamma(\mu)|} \\
& \quad+\frac{\rho}{\pi|\Gamma(\beta+1)|} \sum_{k=1}^{\infty} \frac{\left|\Gamma\left(\mu+\beta+1+\frac{k}{\rho}\right)\right|}{\left|\Gamma\left(\mu+\frac{k}{\rho}\right)\right|}|z|^{k}|\zeta|^{k} \\
& \quad \leq \operatorname{const}\left(a_{1}, a_{2}, A, b_{1}, b_{2}, B, \rho\right) \\
& \quad+\operatorname{const}\left(a_{1}, a_{2}, A, b_{1}, b_{2}, B, \rho\right) \sum_{k=1}^{\infty} \frac{\left(\frac{k}{\rho}\right)^{R e \mu+R e \beta+1+\frac{k}{\rho}-\frac{1}{2}} \cdot e^{-\frac{k}{\rho}}}{\left(\frac{k}{\rho}\right)^{R e \mu+\frac{k}{\rho}-\frac{1}{2}} \cdot e^{-\frac{k}{\rho}}}|z|^{k}|\zeta|^{k} \\
& \quad=\operatorname{const}\left(a_{1}, a_{2}, A, b_{1}, b_{2}, B, \rho\right) \\
& \quad+\operatorname{const}\left(a_{1}, a_{2}, A, b_{1}, b_{2}, B, \rho\right) \sum_{k=1}^{\infty} k^{R e \beta+1}|z|^{k}|\zeta|^{k} \\
& \quad \leq \operatorname{const}\left(a_{1}, a_{2}, A, b_{1}, b_{2}, B, \rho\right) \\
& \quad+\operatorname{const}\left(a_{1}, a_{2}, A, b_{1}, b_{2}, B, \rho\right) \sum_{k=1}^{\infty} k^{a_{2}+1}|z|^{k}|\zeta|^{k} .
\end{aligned}
$$

From here (4) follows. As to (5), it directly follows from the estimation

$$
k^{\operatorname{Re} \beta+1} \asymp \frac{\Gamma(k+R e \beta+2)}{\Gamma(\operatorname{Re} \beta+2) \Gamma(k+1)}
$$


and binomial expansion

$$
\frac{1}{(1-x)^{s}}=\sum_{k=0}^{\infty} \frac{\Gamma(k+s)}{\Gamma(s) \Gamma(k+1)} \cdot x^{k} .
$$

Corollary. For a fixed $z \in \mathbb{D}$ and $\zeta \in \overline{\mathbb{D}}$, the kernel $S_{\beta, \rho, \varphi}(z, \zeta)$ is holomorphic in $\beta$ and $\varphi$ with $\operatorname{Re} \beta>-1$ and $\operatorname{Re} \varphi>-1$.

Theorem 5. Assume that $1 \leq p<\infty, \rho>0, \alpha>-1, \gamma>-1$ and $f \in L_{\alpha, \rho, \gamma}^{p}(\mathbb{D})$. Then there exists a positive function $\Phi(\zeta) \in L^{1}(\mathbb{D})$ such that

$$
\left.\left|f(\zeta)\left(1-|\zeta|^{2 \rho}\right)^{\beta}\right| \zeta\right|^{2 \varphi} S_{\beta, \rho, \varphi}(z, \zeta) \mid \leq \Phi(\zeta), \quad \zeta \in \mathbb{D}
$$

uniformly in $z \in K \Subset \mathbb{D}$ and in $\beta$ and $\varphi$ satisfying the conditions

$$
\begin{aligned}
& \alpha=a_{1} \leq \operatorname{Re} \beta \leq a_{2}, \quad|\operatorname{Im} \beta| \leq A, \\
& \gamma=b_{1} \leq \operatorname{Re} \varphi \leq b_{2}, \quad|\operatorname{Im} \varphi| \leq B
\end{aligned}
$$

when $p=1$ and the conditions

$$
\begin{aligned}
& \frac{\alpha+1}{p}-1<a_{1} \leq \operatorname{Re} \beta \leq a_{2}, \quad|\operatorname{Im} \beta| \leq A, \\
& \frac{\gamma+1}{p}-1<b_{1} \leq \operatorname{Re} \varphi \leq b_{2}, \quad|\operatorname{Im} \varphi| \leq B,
\end{aligned}
$$

when $p>1$.

Proof. Note that under the assumptions of the theorem, $\left|S_{\beta, \rho, \varphi}(z, \zeta)\right| \leq$ const $\left(a_{1}, a_{2}, A, b_{1}, b_{2}, B, \rho, K\right)<+\infty, \zeta \in \overline{\mathbb{D}}$ (see (5)). If $p=1$, then

$$
\begin{array}{r}
\left.\left|f(\zeta) \cdot\left(1-|\zeta|^{2 \rho}\right)^{\beta} \cdot\right| \zeta\right|^{2 \varphi} \cdot S_{\beta, \rho, \varphi}(z, \zeta) \mid \leq \text { const } \cdot|f(\zeta)| \cdot\left(1-|\zeta|^{2 \rho}\right)^{R e \beta} \cdot|\zeta|^{2 \operatorname{Re} \varphi} \\
\leq \text { const } \cdot|f(\zeta)| \cdot\left(1-|\zeta|^{2 \rho}\right)^{\alpha} \cdot|\zeta|^{2 \gamma}=\Phi(\zeta) \in L^{1}(\mathbb{D})
\end{array}
$$

while for $p>1$ we can write

$$
\begin{aligned}
& \left.\left|f(\zeta) \cdot\left(1-|\zeta|^{2 \rho}\right)^{\beta} \cdot\right| \zeta\right|^{2 \varphi} \cdot S_{\beta, \rho, \varphi}(z, \zeta) \mid \\
& \quad \leq \text { const } \cdot|f(\zeta)| \cdot\left(1-|\zeta|^{2 \rho}\right)^{\operatorname{Re} \beta} \cdot|\zeta|^{2 \operatorname{Re} \varphi} \\
& \quad \leq \text { const } \cdot|f(\zeta)| \cdot\left(1-|\zeta|^{2 \rho}\right)^{a_{1}} \cdot|\zeta|^{2 b_{1}} \\
& \quad=\text { const } \cdot|f(\zeta)| \cdot\left(1-|\zeta|^{2 \rho}\right)^{\frac{\alpha}{p}} \cdot|\zeta|^{\frac{2 \gamma}{p}} \cdot\left(1-|\zeta|^{2 \rho}\right)^{a_{1}-\frac{\alpha}{p}} \cdot|\zeta|^{2 b_{1}-\frac{2 \gamma}{p}}=\Phi(\zeta) .
\end{aligned}
$$

It remains to show that $\Phi(\zeta) \in L^{1}(\mathbb{D})$. We have 


$$
\begin{aligned}
\iint_{\mathbb{D}} \Phi(\zeta) d m(\zeta) \leq \mathrm{const} & \left(\iint_{\mathbb{D}}|f(\zeta)|^{p}\left(1-|\zeta|^{2 \rho}\right)^{\alpha}|\zeta|^{2 \gamma} d m(\zeta)\right)^{1 / p} \\
& \left(\iint_{\mathbb{D}}\left(1-|\zeta|^{2 \rho}\right)^{q\left(a_{1}-\frac{\alpha}{p}\right)}|\zeta|^{2 q\left(b_{1}-\frac{\gamma}{p}\right)} d m(\zeta)\right)^{1 / q}
\end{aligned}
$$

and the convergence of the second integral follows from the conditions of the theorem.

\section{Weighted integral representations for the main classes of holomorphic and harmonic functions}

Theorem 6. Assume $1 \leq p<\infty, \rho>0, \alpha>-1, \gamma>-1$, complex numbers $\beta$ and $\varphi$ are satisfying the conditions

$$
\operatorname{Re} \beta \geq \alpha, \quad \operatorname{Re} \varphi \geq \gamma
$$

when $p=1$ and the conditions

$$
\operatorname{Re} \beta>\frac{\alpha+1}{p}-1, \quad \operatorname{Re} \varphi>\frac{\gamma+1}{p}-1,
$$

when $p>1$, and put $\mu=\frac{\varphi+1}{\rho}$. Let $f=u+i v \in H_{\alpha, \rho, \gamma}^{p}$. Then the following integral representations hold: for all $z \in \mathbb{D}$

$$
\begin{aligned}
& f(z)=i v(0)+ \\
& \quad \iint_{\mathbb{D}} u(\zeta)\left(2 S_{\beta, \rho, \varphi}(z, \zeta)-\frac{\rho}{\pi} \frac{\Gamma(\mu+\beta+1)}{\Gamma(\beta+1) \Gamma(\mu)}\right)\left(1-|\zeta|^{2 \rho}\right)^{\beta}|\zeta|^{2 \varphi} d m(\zeta)
\end{aligned}
$$

and

$$
\begin{aligned}
& u(z)= \\
& \iint_{\mathbb{D}} u(\zeta)\left(S_{\beta, \rho, \varphi}(z, \zeta)+S_{\beta, \rho, \varphi}(\zeta, z)-\frac{\rho}{\pi} \frac{\Gamma(\mu+\beta+1)}{\Gamma(\beta+1) \Gamma(\mu)}\right)\left(1-|\zeta|^{2 \rho}\right)^{\beta}|\zeta|^{2 \varphi} d m(\zeta)
\end{aligned}
$$


Proof. First of all, note that

$$
S_{\beta, \rho, \varphi}(0, \zeta)=S_{\beta, \rho, \varphi}(z, 0)=\frac{\rho}{\pi} \frac{\Gamma(\mu+\beta+1)}{\Gamma(\beta+1) \Gamma(\mu)}, \quad z \in \mathbb{D}, \quad \zeta \in \overline{\mathbb{D}}
$$

Further, due to Corollary 1, the expressions under the signs of the integrals are holomorphic in $\beta$ and $\varphi, \operatorname{Re} \beta>-1, \operatorname{Re} \varphi>-1$ for fixed $z$ and $\zeta$. Hence, according to Theorem 5 , for a fixed $z \in \mathbb{D}$, the right-hand side of (9) and (10) are also holomorphic in $\beta$ and $\varphi$ when (8) (or (7), depending on $p$ ) is satisfied. Thus, in view of the uniqueness theorem for holomorphic functions in two complex variables, we can additionally suppose (without loss of generality) that $\beta$ and $\varphi$ are real. According to [7, Theorem 4.2],

$$
f(z)=\iint_{\mathbb{D}} f(\zeta) S_{\beta, \rho, \varphi}(z, \zeta)\left(1-|\zeta|^{2 \rho}\right)^{\beta}|\zeta|^{2 \varphi} d m(\zeta), \quad z \in \mathbb{D},
$$

and

$$
\overline{f(0)}=\iint_{\mathbb{D}} \overline{f(\zeta)} S_{\beta, \rho, \varphi}(z, \zeta)\left(1-|\zeta|^{2 \rho}\right)^{\beta}|\zeta|^{2 \varphi} d m(\zeta), \quad z \in \mathbb{D} .
$$

Summation of 12 and 113 yields

$$
f(z)+\overline{f(0)}=\iint_{\mathbb{D}} 2 u(\zeta) S_{\beta, \rho, \varphi}(z, \zeta)\left(1-|\zeta|^{2 \rho}\right)^{\beta}|\zeta|^{2 \varphi} d m(\zeta)
$$

According to 12 and 11 ,

$$
f(0)=\iint_{\mathbb{D}} f(\zeta) \frac{\Gamma(\mu+\beta+1)}{\Gamma(\beta+1) \Gamma(\mu)} \frac{\rho}{\pi}\left(1-|\zeta|^{2 \rho}\right)^{\beta}|\zeta|^{2 \varphi} d m(\zeta), \quad z \in \mathbb{D} .
$$

Hence,

$$
u(0)=\iint_{\mathbb{D}} u(\zeta) \frac{\Gamma(\mu+\beta+1)}{\Gamma(\beta+1) \Gamma(\mu)} \frac{\rho}{\pi}\left(1-|\zeta|^{2 \rho}\right)^{\beta}|\zeta|^{2 \varphi} d m(\zeta), \quad z \in \mathbb{D} .
$$

Combination of (14) and (15) immediately gives (9).

Further, taking the real parts in (14) we get

$$
u(z)=\iint_{\mathbb{D}} u(\zeta)\left(S_{\beta, \rho, \varphi}(z, \zeta)+S_{\beta, \rho, \varphi}(\zeta, z)\right)\left(1-|\zeta|^{2 \rho}\right)^{\beta}|\zeta|^{2 \varphi} d m(\zeta)-u(0) .
$$

The formulas (16) and (15) together establish 10. 
Theorem 7. Assume $1 \leq p<\infty, \rho>0, \alpha>-1, \gamma>-1$, complex numbers $\beta$ and $\varphi$ are satisfying the conditions

$$
\operatorname{Re} \beta \geq \alpha, \quad \operatorname{Re} \varphi \geq \gamma
$$

when $p=1$ and the conditions

$$
\operatorname{Re} \beta>\frac{\alpha+1}{p}-1, \quad \operatorname{Re} \varphi>\frac{\gamma+1}{p}-1,
$$

when $p>1$, and put $\mu=(\varphi+1) / \rho$. For each $u \in h_{\alpha, \rho, \gamma}^{p}(\mathbb{D})$, the representation (10) holds.

Proof. Repeating the argument from the proof of Theorem 6, we can assume additionally, that $\beta \in \mathbb{R}, \varphi \in \mathbb{R}$ and $\beta>0$.

Since $u$ is a harmonic function, there exists a holomorphic function $f$ in $\mathbb{D}$ such that $u=R e f$ in $\mathbb{D}$. Fix an arbitrary $z_{0} \in \mathbb{D}$ and denote $f_{r}(\zeta)=f(r \zeta)$. Obviously, $f_{r} \in H_{\alpha, \rho, \gamma}^{p}(\mathbb{D})$. As $u(r \zeta)=\operatorname{Re} f(r \zeta)$, from 10$)$ we obtain

$$
\begin{array}{r}
u\left(r z_{0}\right)=\iint_{\mathbb{D}} u(r \zeta)\left(S_{\beta, \rho, \varphi}\left(z_{0}, \zeta\right)+S_{\beta, \rho, \varphi}\left(\zeta, z_{0}\right)-\frac{\rho}{\pi} \frac{\Gamma(\mu+\beta+1)}{\Gamma(\beta+1) \Gamma(\mu)}\right) \\
\left(1-|\zeta|^{2 \rho}\right)^{\beta}|\zeta|^{2 \varphi} d m(\zeta) \\
=\iint_{|\zeta|<r} u(\zeta)\left(S_{\beta, \rho, \varphi}\left(z_{0}, \frac{\zeta}{r}\right)+S_{\beta, \rho, \varphi}\left(\frac{\zeta}{r}, z_{0}\right)-\frac{\rho}{\pi} \frac{\Gamma(\mu+\beta+1)}{\Gamma(\beta+1) \Gamma(\mu)}\right) \\
\quad\left(1-\frac{|\zeta|^{2 \rho}}{r^{2 \rho}}\right)^{\beta} \frac{|\zeta|^{2 \varphi}}{r^{2 \varphi}} \frac{d m(\zeta)}{r^{2}} \\
=\iint_{\mathbb{D}} u(\zeta) \chi_{r}(\zeta)\left(S_{\beta, \rho, \varphi}\left(z_{0}, \frac{\zeta}{r}\right)+S_{\beta, \rho, \varphi}\left(\frac{\zeta}{r}, z_{0}\right)-\frac{\rho}{\pi} \frac{\Gamma(\mu+\beta+1)}{\Gamma(\beta+1) \Gamma(\mu)}\right) \\
\quad\left(1-\frac{|\zeta|^{2 \rho}}{r^{2 \rho}}\right)^{\beta} \frac{|\zeta|^{2 \varphi}}{r^{2 \varphi}} \frac{d m(\zeta)}{r^{2}}=I_{r},
\end{array}
$$

where $\chi_{r}(\zeta)$ is the characteristic function of the disc $\{\zeta:|\zeta|<r\}$ and $I_{r}$ stands for the right-most integral in the expression above. Assume also that $0<r_{0} \leq r<1$ for some $r_{0}$. We intend to let $r \uparrow 1$ in the both sides of

$$
u\left(r \cdot z_{0}\right)=I_{r}
$$

As a result, at the left-hand side of (17) we will obtain $u\left(z_{0}\right)$, while the right hand-side of (17) will coincide with the one in (10) for $z=z_{0}$.

Hence, it remains to show that such passage of the limit is legitimate. To do so, we will use the Lebesgue dominated convergence theorem. Using 
(5) and the assumption $\beta>0$, for the expression under the sign of integral $I_{r}$, we obtain

$$
\begin{aligned}
& \mid u(\zeta) \chi_{r}(\zeta)\left(S_{\beta, \rho, \varphi}\left(z_{0}, \frac{\zeta}{r}\right)+S_{\beta, \rho, \varphi}\left(\frac{\zeta}{r}, z_{0}\right)-\frac{\rho}{\pi} \frac{\Gamma(\mu+\beta+1)}{\Gamma(\beta+1) \Gamma(\mu)}\right) \\
& \qquad\left(1-\frac{|\zeta|^{2 \rho}}{r^{2 \rho}}\right)^{\beta} \frac{|\zeta|^{2 \varphi}}{r^{2 \varphi}} \frac{1}{r^{2}} \mid \\
& \leq \frac{|u(\zeta)|}{r^{2 \rho \beta+2 \varphi+2}}\left|\chi_{r}(\zeta)\right|\left(r^{2 \rho}-|\zeta|^{2 \rho}\right)^{\beta}|\zeta|^{2 \varphi}\left(\frac{\text { const }}{\left(1-\left|z_{0}\right|\right)^{\beta+2}}+\frac{\rho}{\pi} \frac{\Gamma(\mu+\beta+1)}{\Gamma(\beta+1) \Gamma(\mu)}\right) \\
& \leq \frac{\text { const }}{r_{0}^{2 \rho \beta+2 \varphi+2}}|u(\zeta)|\left(1-|\zeta|^{2 \rho}\right)^{\beta}|\zeta|^{2 \varphi}=\text { const }|u(\zeta)|\left(1-|\zeta|^{2 \rho}\right)^{\beta}|\zeta|^{2 \varphi} \equiv \psi(\zeta) .
\end{aligned}
$$

We want to show that $\psi(\zeta) \in L^{1}(\mathbb{D})$, which is equivalent to show that $|u(\zeta)|\left(1-|\zeta|^{2 \rho}\right)^{\beta}|\zeta|^{2 \varphi} \in L^{1}(\mathbb{D})$. For $p=1$, we have

$$
|u(\zeta)|\left(1-|\zeta|^{2 \rho}\right)^{\beta}|\zeta|^{2 \varphi} \leq|u(\zeta)|\left(1-|\zeta|^{2 \rho}\right)^{\alpha}|\zeta|^{2 \gamma} \in L^{1}(\mathbb{D})
$$

If $p>1$, using Holder inequality, we get

$$
\begin{aligned}
\iint_{\mathbb{D}}|u(\zeta)| & \left(1-|\zeta|^{2 \rho}\right)^{\beta}|\zeta|^{2 \varphi} d m(\zeta) \\
& =\iint_{\mathbb{D}}|u(\zeta)|\left(1-|\zeta|^{2 \rho}\right)^{\beta-\frac{\alpha}{p}}|\zeta|^{2 \varphi-\frac{2 \gamma}{p}}\left(1-|\zeta|^{2 \rho}\right)^{\frac{\alpha}{p}}|\zeta|^{\frac{2 \gamma}{p}} d m(\zeta) \\
& \left.\leq \iiint_{\mathbb{D}}|u(\zeta)|^{p}\left(1-|\zeta|^{2 \rho}\right)^{\alpha}|\zeta|^{2 \gamma} d m(\zeta)\right)^{1 / p} \\
& \left(\iint_{\mathbb{D}}\left(1-|\zeta|^{2 \rho}\right)^{q\left(\beta-\frac{\alpha}{p}\right)}|\zeta|^{2 q\left(\varphi-\frac{\gamma}{p}\right)} d m(\zeta)\right)^{1 / q} .
\end{aligned}
$$

The convergence of the last integral follows from the conditions of the theorem.

Remark. In [9]-[13], one can find various interesting results relating to the weighted integral representations of harmonic functions.

\section{References}

[1] W. Wirtinger, ber eine minimumaufgabe im gebiet der analytischen functionen, Monatshefte fur Math. und Phys. 39(1932), pp. 377-384. https://doi.org/10.1007/bf01699078 
[2] S. Bergman, ber unendliche hermitische formen, die zu einem bereiche gehren, nebst anwendungenauf fragen der abbildung durch funktionen von zwei komplexen vernderlichen, Math. Zeit. 29(1929), pp. 641-677. https://doi.org/10.1007/bf01180554

[3] M. M. Djrbashian, On the representability of certain classes of functions meromorphic in the unit disc, Dokl. Akad. Nauk ArmSSR 3(1945), no. 1, pp.3-9 (in Russian).

[4] M. M. Djrbashian, On the problem of representability of analytic functions, Soobshch. Inst. Matem. Mekh. Akad. Nauk ArmSSR 2(1948), pp. 3-40 (in Russian).

[5] A. E. Djrbashian and F. A. Shamoyan, Topics in the theory of $A_{\alpha}^{p}$ spaces, Teubner-Texte zur Math. 105(1988), Leipzig.

[6] M. M. Djrbashian, Weighted integral representations of smooth or holomorphic functions in the unit disc and in the complex plane, J. Contemp. Math. Analysis 28(1993), no. 4, pp.1-27.

[7] F. V. Hayrapetyan, Weighted integral representations of holomorphic functions in the unit disc by means of Mittag-Leffler type kernels, Proc. NAS RA Math. 55(2020), no. 4, pp. 15-30. https://doi.org/10.3103/s1068362320040032

[8] E. Titchmarsh, The Theory of Functions, Nauka, Moscow, 1980 (in Russian).

[9] M. Andersson, Formulas for the $L^{2}$-minimal solutions of the $\partial \bar{\partial}$ equation in the unit ball of $\mathbb{C}^{N}$, Math. Scand. 56(1985), pp. 43-69. https://doi.org/10.7146/math.scand.a-12087

[10] A. H. Karapetyan, Bounded projections in weighted function spaces in a generalized unit disc, Annales Polonici Mathematici 62(1995), no. 3, pp. 193-218. https://doi.org/10.4064/ap-62-3-193-218

[11] K. L. Avetisyan, Bounded projections on mixed norm harmonic spaces, Dokl. NAN Armenii 101 (2001), no. 3, pp. 211-215 (in Russian).

[12] K. L. Avetisyan, Continuous inclusions and Bergman type operators in n-harmonic mixed norm spaces on the polydisc, J. Math. Anal. Appl. 291 (2004), no. 2, pp. 727-740. https://doi.org/10.1016/j.jmaa.2003.11.039

[13] N. T. Gapoyan, Bergman type operators on the spaces of functions in the ball from $\mathbb{C}^{n}$, Candidate Dissertation, Yerevan, 2017 (in Russian). 
Feliks Hayrapetyan

Yerevan State University

1 Alex Manoogian St, 0025 Yerevan, Armenia.

feliks.hayrapetyan1995@gmail.com

Please, cite to this paper as published in

Armen. J. Math., V. 13, N. 5(2021), pp. 1111

https://doi.org/10.52737/18291163-2021.13.5-1-11 